\title{
Effects of equation of state on nuclear suppression and the initial entropy density of quark gluon plasma
}

\author{
Surasree Mazumder and Jan-e Alam \\ Theoretical Physics Division, Variable Energy Cyclotron Centre, 1/AF, Bidhan Nagar, Kolkata - 700064
}

(Dated: June 16, 2018)

\begin{abstract}
We study the effects of the equation of state on the nuclear suppression of heavy flavours in quark gluon plasma and estimate the initial entropy density of the system produced at the highest RHIC energy. For this purpose we have used the experimental data on the charged particle multiplicity and the nuclear suppression of single electron spectra originating from the semi-leptonic decays of open charm and beauty mesons. We have used inputs from lattice QCD to minimize the model dependence of the results. We obtain the value of the initial entropy density which varies from 20 to $59 / \mathrm{fm}^{3}$ depending on the value of the velocity of sound that one uses for the analysis. Our investigation leads to a conservative value of the initial entropy density $\sim 20 / \mathrm{fm}^{3}$ with corresponding initial temperature $\sim 210 \mathrm{MeV}$ well above the value of the transition temperature predicted by lattice QCD.
\end{abstract}

PACS numbers: 12.38.Mh,25.75.-q,24.85.+p,25.75.Nq

A thermalized system of quarks and gluons, called quark gluon plasma (QGP) is expected to be formed in the collisions of two nuclei at ultra-relativistic energies [1]. Rigorous experimental and theoretical efforts are on to create and characterize this novel, deconfined phase of quarks and gluons. Lattice QCD (LQCD) calculations indicate that at a temperature $\sim 175 \mathrm{MeV}$ the entropy density $(s)$ of the hadronic matter rises significantly due to the release of colour degrees of freedom which are confined within the hadrons at zero temperature. Therefore, it is of foremost importance to determine the value of the initial entropy density $\left(s_{i}\right)$ / initial temperature $\left(T_{i}\right)$ for the system formed in nuclear collisions at Relativistic Heavy Ion Collider (RHIC) and Large Hadron Collider (LHC) and assess whether the system is formed in colour deconfined phase or not. The focus of the present study is to estimate $s_{i}$ or $T_{i}$ of the system formed at $\mathrm{Au}+\mathrm{Au}$ collisions at $\sqrt{s_{\mathrm{N} N}}=200 \mathrm{GeV}$. For this purpose we strict to take inputs from experimental data and LQCD calculations to minimize the model dependence of the outcome of the present analysis.

One of the possible way to estimate the value of the initial entropy density is the extrapolation of the measured (final) observables backward in time through a suitable dynamical model. In absence of viscous loss the time reversal symmetry of the system is valid, therefore, the measured multiplicity at the freeze-out of the system can be used to estimate $s_{i}$. The $s_{i}$ and the thermalization time $\left(\tau_{i}\right)$ are constrained by the measured (final) hadron multiplicity $(d N / d y)$ by the following relation [2]:

$$
s_{i} \tau_{i}=\kappa \frac{1}{A_{\perp}} \frac{d N}{d y}
$$

where $A_{\perp}$ is the transverse area of the system can be determined from the collision geometry and $\kappa$ is a known constant ( $=3.7$ for massless bosons like pions). The value of $d N / d y$ which is connected to $s_{i}$ through Eq. 1 is readily available for different collision centralities [1]. In Eq. 1 there are two unknown quantities, $\tau_{i}$ and $s_{i}$ both of which can not be determined from a single equation involving a single measured the $d N / d y$. Therefore, we choose another experimentally measured quantity the nuclear suppression of heavy quarks (HQ), $R_{\mathrm{AA}}[3$, , 4], which is sensitive to the initial condition and hence is very useful to estimate $s_{i}$.

The advantage of choosing the heavy flavour are (a) they are produced in early hard collisions and hence can witness the entire evolution of the QGP and (b) HQ are Boltzmann suppressed at the temperature range expected to be achieved in heavy ion collisions at RHIC, therefore, the HQ do not determine the bulk features of the QGP. The magnitude of $R_{\mathrm{AA}}$ depends on the amount of drag the heavy quark faces during its propagation through QGP. The heavy quarks being the witness of the early condition and the drag (and diffusion) coefficient is a temperature dependent quantity that makes the $R_{\mathrm{A} A}$ a good probe for the measurement of initial temperature.

Earlier various attempts have been made to explain the experimental results on $R_{A A}$ - some of these are addition of non-perturbative effects from the quasi-hadronic bound state [5], three-body scattering process [ [6] , dissociation of open heavy flavoured mesons by the thermal partons [7] and inclusion of temperature dependent strong coupling [8].

We briefly outline the procedure of evaluating the $R_{A A}$ for single electrons originating from the semi-leptonic decays of heavy mesons produced from the fragmentation of the HQ. The HQ while propagating through the QGP dissipates energy in the medium and hence its momentum gets attenuated. The magnitude of the momentum degradation gets reflected in the experimentally measured quantity, $R_{A A}$ mentioned above. Theoretically the momentum evolution of the HQ in the expanding QGP background can be described by using Fokker Planck Equation (FPE) [9 19]. The evolution of the probe i.e. 
the HQ is described by the FPE:

$$
\frac{\partial f}{\partial t}=\frac{\partial}{\partial p_{i}}\left[A_{i}(\mathbf{p}) f+\frac{\partial}{\partial p_{j}}\left[B_{i j}(\mathbf{p}) f\right]\right],
$$

where

$$
A_{i}=\int d^{3} \mathbf{k} w(\mathbf{p}, \mathbf{k}) k_{i}=\gamma p_{i}
$$

and

$$
B_{i j}=\frac{1}{2} \int d^{3} \mathbf{k} w(\mathbf{p}, \mathbf{k}) k_{i} k_{j}=D \delta_{i j} .
$$

$\gamma$ and $\mathrm{D}$ are called the drag and diffusion coefficients, contain the interaction of the probe with the medium. To solve the FPE we need to supply the drag and diffusion coefficients and the initial HQ (charm and beauty) momentum distributions.

There are two main processes through which the HQ dissipates energy in the QGP: (i) energy dissipation can take place due to the elastic collisions of the HQ with the quarks, antiquarks and gluons in the thermal bath, (ii) the radiative process due to which the $\mathrm{HQ}$ emits soft gluons (which subsequently get absorbed in the QGP) due to its interaction with the QGP. The details solution of FPE with temperature and momentum dependent drag and diffusion coefficients including both the processes (i) and (ii) have been discussed in our earlier works [18]. The initial momentum distributions of charm and bottom quarks at RHIC energy $\left(\sqrt{s_{\mathrm{N} N}}=200 \mathrm{GeV}\right)$ have been taken from MNR code [20]. The method of solving the FPE numerically with temperature and momentum dependent transport coefficients including other issues are discussed in [18], therefore we do not repeat those details here.

Classically the induced radiation takes place due to the jiggling motion of the propagating particle in the medium. Since the heavier particle jiggle less consequently induced energy loss is expected to be smaller [dead cone effect [21] (see also [22, 23])] for HQ compared to light particles. However, the experimental data from RHIC indicates similar amount of energy loss by heavy quarks and light partons in the measured kinematic range. Various reasons like the anomalous mass dependence of the radiative process due to the finite size of the QGP 24], development of dead cone due to high virtuality of the partons resulting from the dismantling of colour fields during the initial hard collisions [25] have been proposed as reasons for this observation. The authors in 26] concluded that the reduction in the energy loss of HQ due to radiative process is due to the dead cone effect but it is fair to mention that the issue is yet to be settled.

The other effect which influences the radiative loss is the Landau-Pomeranchuk-Migdal(LPM) effect. This effect originate due to the interplay between two time scales of the system [27]: the formation time $\left(\tau_{F}\right)$ and the mean scattering time $\left(\tau_{s c}\right)$ of the gluon radiated from the HQ. LPM effect imposes certain constrain on the phase space of the emitted gluon [28, 29]. Both the dead cone and the LPM effects have been taken in to account in evaluating the drag and diffusion coefficients in the present work (see [18, 19] for details).

Now we discuss the QGP background with which the HQ interacts. The equation of state (EoS) plays a crucial role in describing the space time evolution of the expanding QGP from the initial state to the quark-hadron transition point. We use boost invariant hydrodynamic model [30] with the LQCD calculation EoS [31] for the space time description of the matter. The velocity of sound $\left(c_{s}\right)$ as obtained in [31] from LQCD calculations shows a significant variation with temperature (Fig. 1). It starts with a very low value of $c_{s}^{2}$ at $T \sim T_{c}$ and then increases with $T$ to reach the maximum value $\left(c_{s}^{2}=1 / 3\right)$ corresponding to the value of a massless ideal gas. The EoS for almost baryon free QGP expected at RHIC energy is take as: $P=c_{s}^{2} \epsilon$. The EoS sets the expansion time scale for the system as $\tau_{\text {exp }} \sim[(1 / \epsilon) d \epsilon / d \tau]^{-1} \sim \tau /\left(1+c_{s}^{2}\right)$ indicating the fact that lower value of $c_{s}$ makes the expansion time scale longer i.e. the rate of expansion slower. Therefore, for given values of $T_{i}$ and $T_{c}$ the life time of the QGP will be longer for smaller $c_{s}$. The value of $T_{c}$ is fixed at $175 \mathrm{MeV}$.

We solve the FPE numerically [18] for momentum dependent drag and diffusion coefficients to get the charm and beauty quarks momentum distribution. The solution then convoluted with the fragmentation functions [32] to obtain the transverse momentum distribution of the $D$ and $B$ mesons which subsequently decay to create leptons 33,34$]$.

In the same way the lepton spectra from the heavy flavours produced in p-p collisions can be calculated from the charm and beauty quark distributions which enter as initial conditions to the FPE. The solution of FPE contains the effects of drag (quenching) on the HQ where as the the initial distributions of HQ does not contain any such effects, therefore the ratio of these two quantities, the nuclear suppression factor, $R_{A A}$ act as a marker for the medium. This is observed experimentally through the depletion of $R_{A A}$ at high transverse momentum $\left(p_{T}\right)$.

As discussed above for larger $c_{s}$ the expansion time scale is shorter i.e. the QGP life time is smaller. consequently the HQ spends less time in the QGP which ultimately leads to less suppression of the single electron spectra originating from the decays of HQ. Therefore, we take the following strategy to obtain the allowed range of initial temperature. We take the highest possible value of $c_{s}^{2}(=1 / 3)$ for the space-time description of the flowing QGP background, in the present approach this will lead to the maximum value of $T_{i}$. In this case the $\mathrm{HQ}$ will spend the lesser amount of time in the QGP. Therefore, to achieve the experimentally measured $R_{\mathrm{A} A}$ one will need larger drag or in other word larger initial temperature. The results for $c_{s}^{2}=1 / 3$ is displayed in Fig. 2. The value of $T_{i}$ obtained from the analysis for this case is $300 \mathrm{MeV}$, the corresponding value of $s_{i}=2 \pi^{2} g_{\mathrm{e} f f} T_{i}^{3} / 45 \sim 59 / \mathrm{fm}^{3}$. 
The value of $g_{\mathrm{e} f f} \sim 38$ is extracted from the variation of $s / T^{3}$ with $T$ provided by the LQCD calculations [31].

In Fig. 3 results for $c_{s}^{2}=1 / 4$ are depicted. For $c_{s}^{2}=1 / 4$ the HQ spend longer time in QGP than for $c_{s}^{2}=1 / 3$. Therefore, with a lower initial temperature, $T_{i}=240$ $\mathrm{MeV}$ and $s_{i} \sim 29.34 / \mathrm{fm}^{3}$, the data can be reproduced.

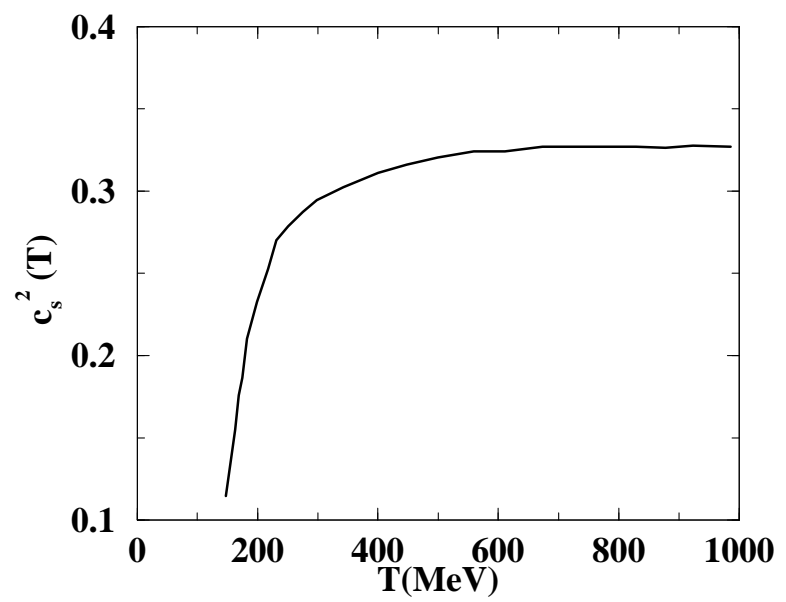

FIG. 1: Velocity of sound squared as a function temperature 31]

For $c_{s}^{2}=1 / 5$ the HQ spend longer time in QGP (compared to the case when $\left.c_{s}^{2}=1 / 4\right)$. Therefore, the observed suppression dictates to reduce the initial temperature. In this case the data is well reproduce with $T_{i}=210$ $\mathrm{MeV}$ and $s_{i} \sim 19.66 / \mathrm{fm}^{3}$.

Further lowering of $c_{s}$ will make the value of $\tau_{i}$ large (for given $d N / d y$ ) enough to contradict other results like the observation of large hadronic elliptic flow which requires small $\tau_{i}$ (see 35] for review). That will also results in lower $T_{i}$ with which it will be difficult explain other experimental results.

For all the theoretical results displayed in Figs. 2, 3 and 4 we have kept the quantity $d N / d y$ constant consequently the value of $\tau_{i}$ changes to $0.6,1.17$ and $1.7 \mathrm{fm} / \mathrm{c}$ for $T_{i}=300,240$ and $210 \mathrm{MeV}$ respectively. The changes in $T_{i}$ is forced by the change in the EoS. In Fig. 5 we show the variation of $T_{i}$ with $c_{s}^{2}$ obtained by constraints imposed by the experimental data on $R_{\mathrm{A} A}$ and $d N / d y$. The value of $T_{i}$ varies from 210 to $300 \mathrm{MeV}$. In this context we compare the value of $T_{i}$ obtained in the work with some of those reported earlier. In Refs. [5] the value of $T_{i}$ is obtained as $\sim 375 \mathrm{MeV}$ from the study of heavy quark suppression. From the simultaneous analysis of light and heavy quarks suppressions in Ref. [36] a value of $T_{i}=400 \mathrm{MeV}$ is obtained. The authors in Ref. [37 39] mentioned the values of the initial gluon rapidity distribution, $d N_{g} / d y$, which may be converted to $T_{i}=290,270$ and 310 respectively.

It is interesting to note that the lowest value of $T_{i}$ obtained from the present analysis is well above the quark-hadron phase transition temperature, indicating

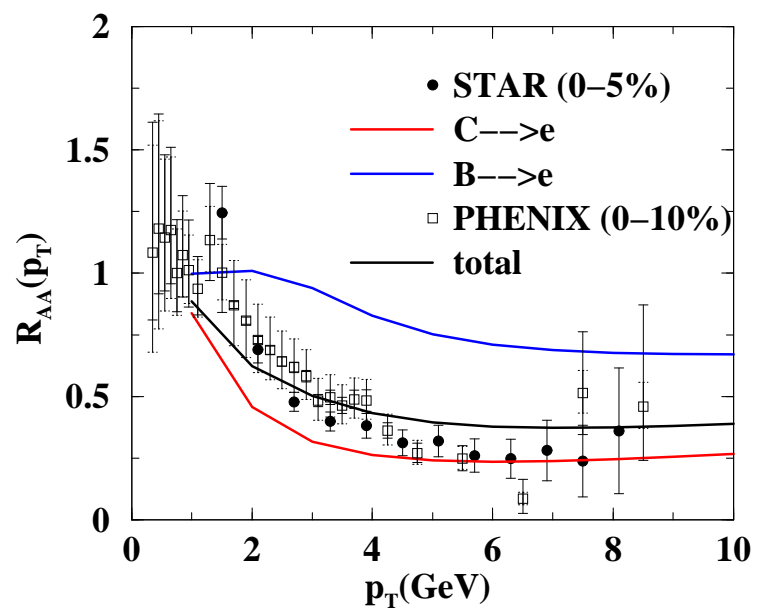

FIG. 2: (colour online) Variation of $R_{A A}$ with $p_{T}$ for $c_{s}^{2}=1 / 3$ and $T_{i}=300 \mathrm{MeV}$.

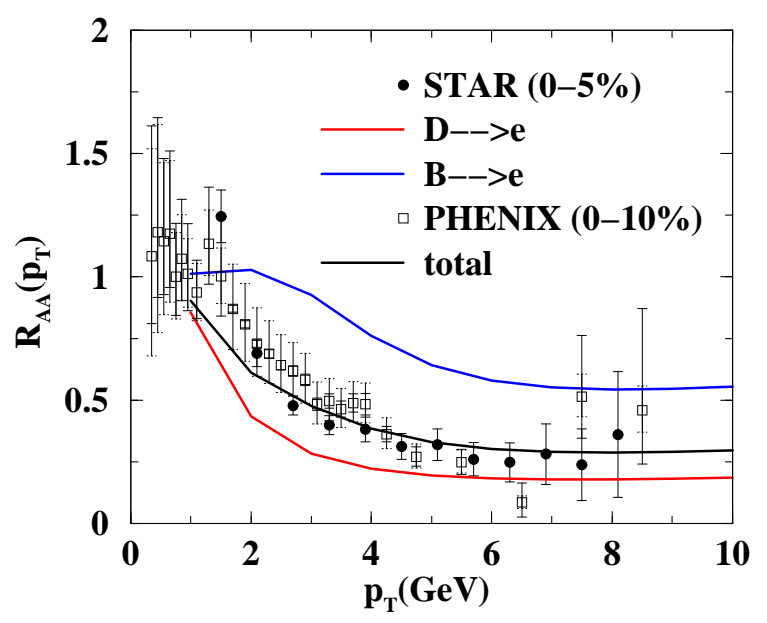

FIG. 3: (colour online) Variation of $R_{A A}$ with $p_{T}$ for for $c_{s}^{2}=$ $1 / 4$ and $T_{i}=240 \mathrm{MeV}$.

the fact that the system formed in $\mathrm{Au}+\mathrm{Au}$ collisions at $\sqrt{s_{\mathrm{N} N}}=200 \mathrm{GeV}$ might be formed in the partonic phase.

In summary, we have studied the effects of the EoS on the suppression of single electrons originating from the decays of heavy flavours produced in $\mathrm{Au}+\mathrm{Au}$ collisions at $\sqrt{s_{\mathrm{N} N}}=200 \mathrm{GeV}$. We found that the initial temperature may vary from 210 to $300 \mathrm{MeV}$ depending on the velocity of sound, which sets the scale for the expansion that one uses. We have used experimental data (charged particle multiplicity and $R_{\mathrm{A} A}$ of heavy flavours) and LQCD results $\left(c_{s}, g_{\mathrm{e} f f}\right.$ etc.) to keep the model dependence minimum. The effects of transverse expansion is neglected here. With the transverse expansion the HQ will (1) travel longer path (2) with diluted density. However, the two competing effects (1) and (2) will have some sort of cancellation due to which our final conclusion may 


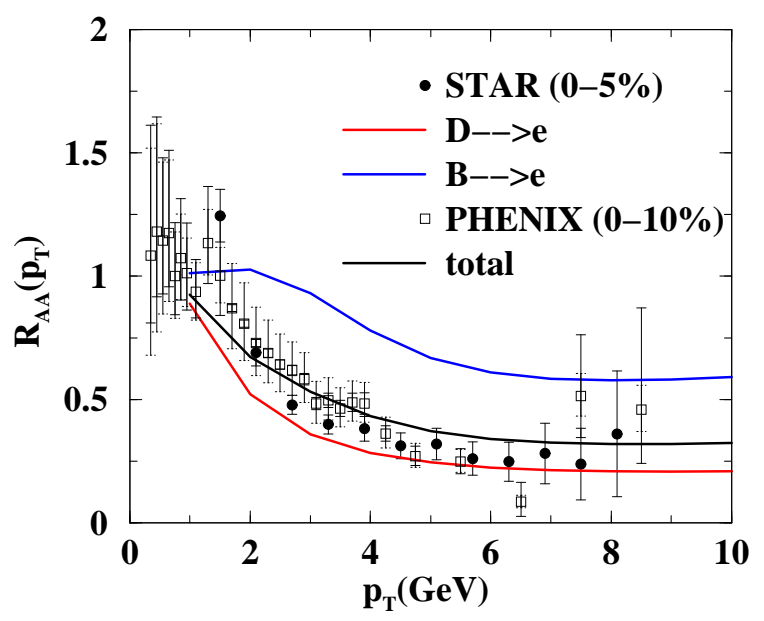

FIG. 4: (colour online) Variation of $R_{A A}$ with $p_{T}$ for $c_{s}^{2}=1 / 5$ and $T_{i}=210 \mathrm{MeV}$.

not get altered.

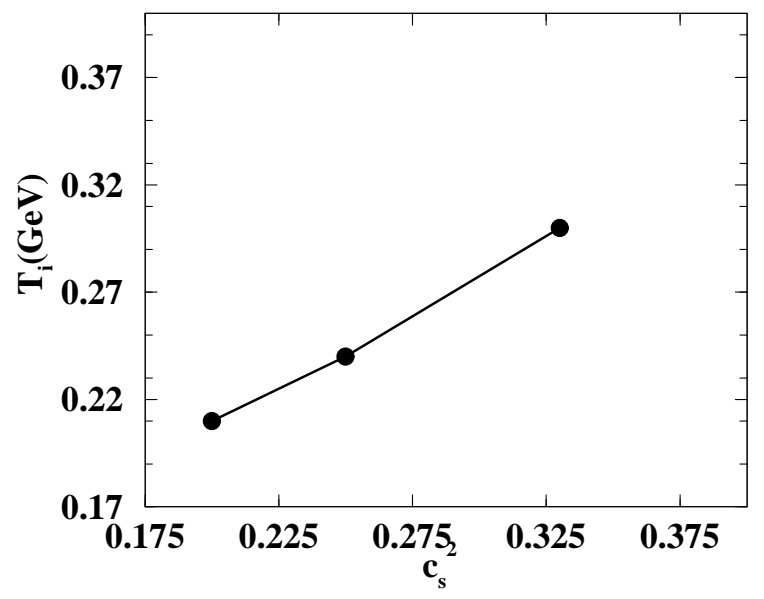

FIG. 5: The variation of $T_{i}$ with $c_{s}^{2}$ for fixed $d N / d y$.
Acknowledgement: SM is grateful to Department of Atomic Energy, Government of India for financial support. We are thankful to Santosh K Das and Trambak Bhattacharyya for useful discussions.
[1] I. Arsene et al. (BRAHMS Collaboration), Nucl. Phys. A 757, 1 (2005); B. B. Back et al. (PHOBOS Collaboration), Nucl. Phys. A 757, 28 (2005); J. Adams et al. (STAR Collaboration), Nucl. Phys. A 757, 102 (2005); K. Adcox et al. (PHENIX Collaboration), Nucl. Phys. A 757, 184,(2005).

[2] R. C. Hwa and K. Kajantie, Phys. Rev. D, 32, 1109 (1985).

[3] B. I. Abeleb et al. (STAR Collaboration), Phys. Rev. Lett. 98, 192301 (2007).

[4] S. S. Adler et al. (PHENIX Collaboration), Phys. Rev. Lett. 96, 032301 (2006).

[5] H. van Hees, M. Mannarelli, V. Greco and R. Rapp, Phys. Rev. Lett. 100, 192301 (2008).

[6] C. M. Ko and W. Liu, Nucl. Phys. A 783, 23c (2007).

[7] A. Adil and I. Vitev, Phys. Lett. B 649, 139 (2007).

[8] P. B. Gossiaux and J. Aichelin, Phys. Rev. C 78, 014904 (2008).
[9] S. Chakraborty and D. Syam, Lett. Nuovo Cim. 41, 381 (1984).

[10] H. van Hees, R. Rapp, Phys. Rev. C,71, 034907 (2005).

[11] S. Turbide, C. Gale, S. Jeon and G. D. Moore, Phys. Rev. C 72, 014906 (2005).

[12] J. Bjoraker and R. Venugopalan, Phys. Rev. C 63, 024609 (2001). 1

[13] B. Svetitsky, Phys. Rev. D 37, 2484( 1988).

[14] J. Alam, S. Raha and B. Sinha, Phys. Rev. Lett. 73, 1895 (1994).

[15] P. Roy, J. Alam, S. Sarkar, B. Sinha and S. Raha, Nucl. Phys. A 624, 687 (1997).

[16] M G. Mustafa and M. H. Thoma, Acta Phys. Hung. A 22, 93 (2005).

[17] P. Roy, A. K. Dutt-Mazumder and J. Alam, Phys. Rev. C 73, 044911 (2006).

[18] S. Mazumder, T. Bhattacharya, J. Alam and S. K. Das, 
arXiv:1106.2615 [nucl-th]; Phys. Rev. C (in press).

[19] S. K. Das, J. Alam and P. Mohanty, Phys. Rev. C 82, 014908 (2010); S. K. Das, J. Alam and P. Mohanty, Phys. Rev. C 80, 054916 (2009); S. K. Das, J. Alam, P. Mohanty and B. Sinha, Phys. Rev. C 81, 044912 (2010).

[20] M. L. Mangano, P. Nason and G. Ridolfi, Nucl. Phys.B 373, 295 (1992).

[21] Y. L. Dokshitzer and D. E. Kharzeev, Phys. Lett. B, 519, 199 (2001).

[22] B. W. Zhang, E. Wang and X.-N. Wang, Phys. Rev. Lett. 93, 072301 (2004).

[23] N. Armesto, A. Dainese, C. A. Salgado and U. A. Wiedemann, Phys. Rev. D 71, 054027 (2005).

[24] B. G Zakharov, JETP Lett. 86, 444(2007); P. Aurenche and B. G. Zakharov, JETP Lett. 90, 237 (2009).

[25] B. Z. Kopeliovich, I. K. Potashnikova, I. Schmidt, Phys. Rev. C 82, 037901(2010)

[26] Roy A. Lacey et al., Phys. Rev. Lett.103,142302(2009)

[27] S. Klein, Rev. Mod. Phys. 71, 1501 (1999)

[28] X. N. Wang, M. Gyulassy and M. Plümer, Phys. Rev. D
51, 3436 (1995).

[29] M. G. Mustafa, D. Pal, D. K. Srivastava and M. H. Thoma, Phys. Lett. B, 428,234(1998).

[30] J. D. Bjorken, Phys. Rev. D 27, 140 (1983).

[31] S. Borsáyi et al., J. High. Ener. Phys 1011, 077 (2010)

[32] C. Peterson et al., Phys. Rev. D 27, 105 (1983).

[33] M. Gronau, C. H. Llewellyn Smith, T. F. Walsh, S. Wolfram and T. C. Yang, Nucl. Phys. B 123, 47 (1977).

[34] A. Ali, Z. Phys. C 1, 25 (1979).

[35] P. Huovinen and P. V. Ruuskanen, Ann. Rev. Nucl. Part. Sci. 56 (2006) 163; D. A. Teaney, arXiv:0905.2433 [nuclth].

[36] G. Qin and A. Majumder, Phys. Rev. Lett. 105, 262301 (2010).

[37] M. Djordjevic, J. Phys. G 32, 333 (2006).

[38] J. Uphoff, O. Fochler, Z. Xu and C. Greiner, Phys. Rev. C 84, 024908(2011).

[39] I. Vitev, A. Adil and H. Van Hees, J. Phys. G 34, 769 (2007). 